\section{Organschaft}

Die Zeitschrift ist amtliches Organ der Bundesanstalt für Materialforschung und -prüfung sowie Organ des Normenausschusses Materialprüfung im DIN

\section{Herausgeber}

BAM Bundesanstalt für Materialforschung und -prüfung DGZfP Deutsche Gesellschaft für Zerstörungsfreie Prüfung

DVM Deutscher Verband für Materialforschung und -prüfung

VDI-Gesellschaft Werkstofftechnik

\section{Aufsatz-Redaktion Berlin}

Dr.-Ing. Dietmar Aurich, Ltd. Dir. u.

Prof. (verantwortlich)

Dr. Manfred P. Hentschel

Dr. Jürgen Sickfeld

Dr. Uta Völkel

BAM, Unter den Eichen 87

D-1000 Berlin 45

Telefon: 0 30/8104-1

Telefax: 0 30/8112029

\section{Redaktion Darmstadt}

Dipl.-Ing. Claudia Treffert

Redaktionsassistentin:

Traudl Erbeldinger

Carl Hanser Verlag

Marburger Straße 13

D-6100 Darmstadt

Telefon: $06151 / 700950$

Telefax: 0 6151/70 0948

Beiträge und Mitteilungen für den redaktionellen Inhalt sind an die Redaktion Darmstadt zu senden. Fachaufsätze (Originalbeiträge) an die Aufsatzredaktion Berlin

Postfach 8604 20, 8000 München 86

Telefon (0 89) 99830-0

Vertrieb: (0 89) 99830-131

Anzeigen: (0 89) 99830-221

Fax (0 89) 984809

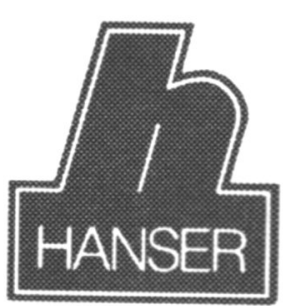

Carl Hanser Verlag

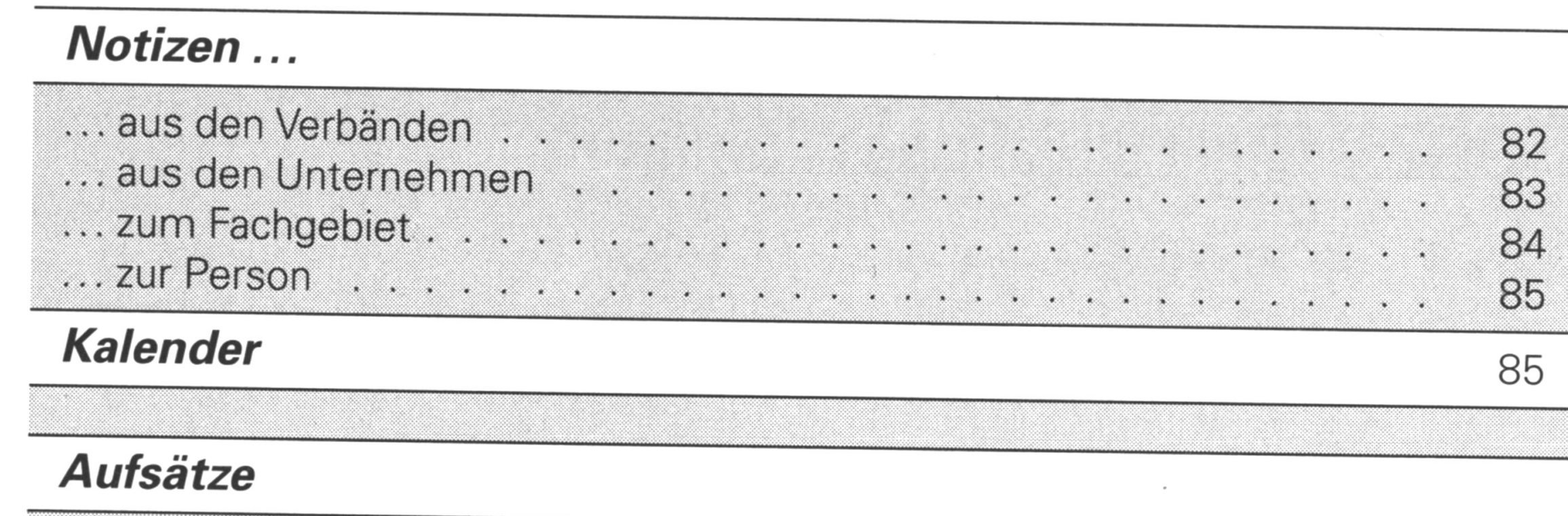

R. Drumm: Ein PC regelt servohydraulische Zylinder

H. Vetters, T. Hirsch, H. R. Stock, P. Mayr: Dünne Hartstoffschichten prüfen

St. Risse, D. Schnapp: Risse in Gläsern optoelektronisch ausmessen

M. Wloka: Wer zertifiziert und akkreditiert in Europa? -

Übersicht über nationale und internationale Organisationen

G. Busse, W. Michaeli, G. Aengenheyster, P. Höck, S. Kempa,

D. Wu, L. Diener: Anisotropien von Kunststoffen:

Produzieren, Messen und Vorhersagen

W. Ricken, W.-J. Becker: Durchmesserunabhängig die Betondeckung messen - Wirbelstrommeßverfahren trennt Parameter

\section{Kurzberichte}

Mechanisch-technologische Prüfungen an Schreibgeräten

Einfache Härtemessung in schwierigen Fällen

88

E-Modul von Magnetspulen bestimmen . . . . . . . . . . . . 89

Photogoniometer für hochwertige Oberflächen . . . . . . . . . 97

Härte mit interaktiver oder automatischer Bildanalyse messen . . . . . . 97

Neuartige Aufnehmer für die Ultraschallprüfung . . . . . . . . . . . 100

Schweißnähte am Bildschirm inspizieren . . . . . . . . . . . . 101

Schichtdicken in line messen ............... . . . 101

Glanz nach Norm geprüft _. . . . . . . . . . . 101

Verbundnetz für Klimaprüfanlagen 101

Berührungslose Dehnungsmessung mit Video-Extensometer . . . . . . 107

Von innen nach außen: Tankböden inspizieren . . . . . . 107

Triaxiale Prüfung kubischer Frostproben . . . . . . . . . . . . 115

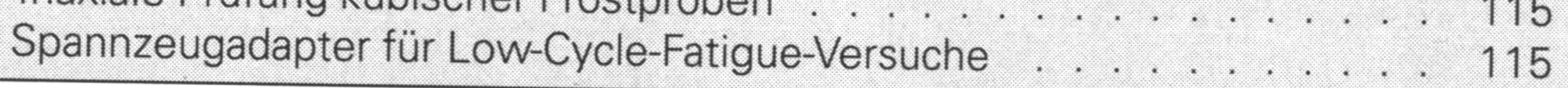

$\begin{array}{ll}\text { Normen und Richtlinien } & 116\end{array}$

$\begin{array}{ll}\text { Bücher } & 116\end{array}$

Report 118

$\begin{array}{ll}\text { Impressum } & 120\end{array}$

\title{
Zur Titelseite
}

Für die sichere Bauteilauslegung ist neben den im quasi-statischen Zugversuch ermittelten Festigkeitskennwerten auch die Kenntnis des Werkstoffverhaltens unter hohen Belastungsgeschwindigkeiten notwendig. Mit der neuentwickelten servohydraulischen Hydropuls ${ }^{\circledR}$-Prüfmaschine VHS 100/20 kann der Einfluß der Belastungsgeschwindigkeit von quasi-statisch bis $20 \mathrm{~m} / \mathrm{s}$ untersucht werden, aber auch Ermüdungsversuche sind durchführbar.

Mit einer maximalen Zerreißkraft von $100 \mathrm{kN}$ und der neuentwickelten prellfreien Schnellzerreißvorrichtung "Schneller Beißer" nimmt die VHS 100/20 eine technologische Spitzenstellung ein.

Ausführliches darüber können Sie auch in der nächsten Ausgabe der MATERIALPRÜFUNG lesen.

${ }^{\circledR}$ Hydropuls ist eingetragenes Warenzeichen der Carl Schenck AG, Darmstadt

\section{Carl Schenck AG}

Landwehrstr. 55

6100 Darmstadt 1

Tel.: (0 6151$)$ 32-2733

Fax: (06151) 323979

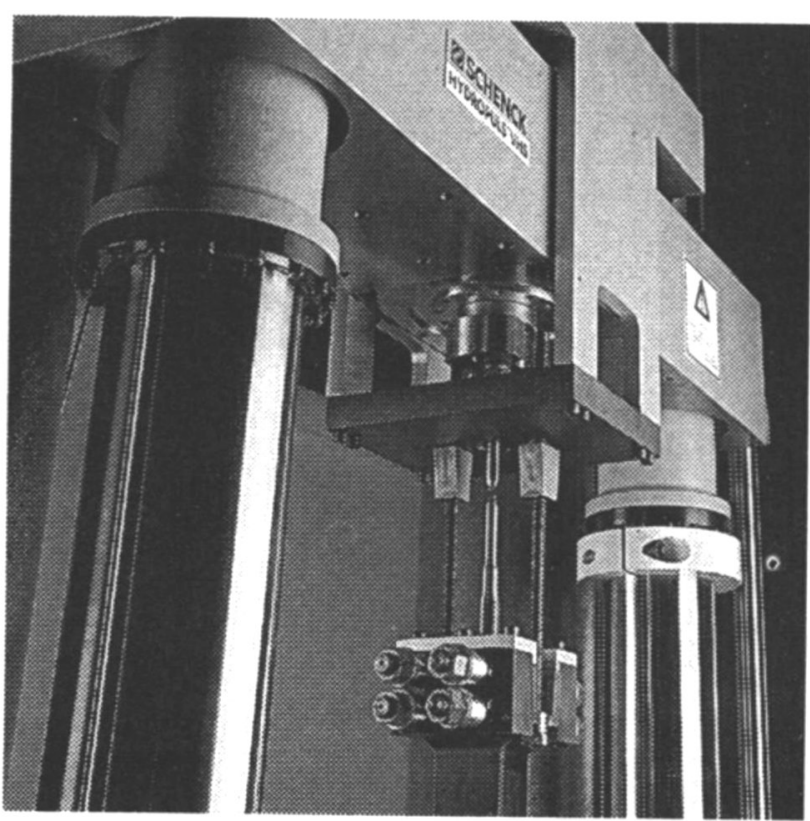

